\title{
Precise epitope determination of the anti-vimentin monoclonal antibody V9
}

\author{
LUCIA TOMIYAMA ${ }^{1,2}$, HIROKI KAMINO $^{1}$, HIROKI FUKAMACHI ${ }^{1}$ and TAKESHI URANO ${ }^{1}$ \\ ${ }^{1}$ Department of Biochemistry, Shimane University Faculty of Medicine, Izumo 693-8501; \\ ${ }^{2}$ Center for The Promotion of Project Research, Shimane University, Matsue 690-8504, Japan
}

Received June 27, 2016; Accepted March 7, 2017

DOI: $10.3892 / \mathrm{mmr} .2017 .7102$

\begin{abstract}
Vimentin is a type III intermediate filament protein that is typically expressed in mesenchymalcells. Overexpression of vimentin is frequently observed in several types of cancer and is often associated with epithelial-to-mesenchymal transition. It was recently reported that the serum vimentin level is significantly elevated in colon and liver tumors. Therefore, a more sensitive vimentin detection system may be useful for cancer screening and early detection. The V9 mouse monoclonal antibody (mAb), which recognizes the human vimentin protein, is widely used in routine pathology to identify mesenchymal cells using immunohistochemical analysis. Although it has been suggested that the epitope of the $\mathrm{V} 9 \mathrm{mAb}$ is located within the C-terminal region of vimentin, the precise amino acid sequence that it recognizes has not yet been identified. In the present study, we constructed several deletion mutants of the vimentin protein and examined their reactivity with the V9 mAb to accurately map its epitope. We confirmed that its epitope resides in the C-terminal region of vimentin, between amino acids 392-466. Additionally, cross-species comparison of amino acid sequence alignment of vimentin, as well as site-directed mutagenesis, revealed that one residue, the asparagine at position 417 , is critical for antibody binding. Using smaller vimentin fragments ranging in length from 9 to 13 residues, each containing this critical asparagine, we determined that the minimal residues required for $\mathrm{V} 9 \mathrm{mAb}$ recognition of human vimentin are the thirteen amino acid residues at positions 411-423 $\left({ }^{411}\right.$ ISLPLPNFSSLNL $\left.{ }^{423}\right)$.
\end{abstract}

Correspondence to: Professor Lucia Tomiyama or Professor Takeshi Urano, Department of Biochemistry, Shimane University Faculty of Medicine, 89-1 Enya-cho, Izumo 693-8501, Japan

E-mail: luciat@med.shimane-u.ac.jp

E-mail: turano@med.shimane-u.ac.jp

Keywords: vimentin, monoclonal antibody, V9, epitope determination

\section{Introduction}

Vimentin is a type III intermediate filament protein that is expressed in cells of mesenchymal origin (1), and is known to function in cell adhesion, migration, and cell signaling (2). Vimentin is a 466 amino acid-long protein composed of three domains: The amino-terminal domain (head domain, 77 residues), the central core (rod domain, 328 residues), and the carboxy-terminal domain (tail domain, 61 residues) (3). The central rod domain mediates coiled-coil dimer formation and these dimers then associate in a staggered fashion to form tetramers, which can assemble end-to-end to form protofilaments $(3,4)$. On the other hand, the disassembly of vimentin filaments is regulated by phosphorylation of serine/threonine residues on the amino-terminal head domain (5). Mitotic phosphorylation of vimentin is important for normal cell division and a defect of vimentin head domain phosphorylation causes microophthalmia and cataracts via aneuploidy and senescence in lens epithelial cells (6).

In recent years, vimentin has been considered as a marker for epithelial-to-mesenchymal transition (EMT), a process in which epithelial cells acquire a mesenchymal migratory phenotype (7). Furthermore, it has been suggested that phosphorylation of vimentin plays an important role in regulating the EMT process. It was reported that Akt binding results in serine phosphorylation of vimentin at amino acid position 39, thereby enhancing the ability of vimentin to induce human soft-tissue sarcoma cell migration and invasion (8). Besides the importance of vimentin phosphorylation for cancer cell migration, overexpression of vimentin has also been reported in various epithelial cancers (9) and correlates with increased tumor growth, invasion and poor prognosis $(10,11)$. Additionally, there is evidence suggesting that vimentin is present in human serum. Sun et al demonstrated that serum vimentin, assayed using an indirect ELISA, is a promising marker in the detection of small liver tumors $(\leq 2 \mathrm{~cm})(12)$. Using affinity proteomics analysis, Bukhari et al recently reported that vimentin expression was higher in the sera of colon cancer patients compared to healthy controls (13). Based on these results, development of a serum test with high sensitivity for the detection of vimentin protein levels is a promising approach for screening and early diagnosis of cancers.

Several antibodies against human vimentin are commercially available and are known to target specific regions of 
the protein. For example, the rod domain is recognized by mouse monoclonal antibody (mAb) 3B4, and the tail domain is recognized by mouse mAb V9 (14). Although mAb V9 was established in 1984 (15) and is widely utilized in both research and diagnostics, the exact amino acid sequence recognized by V9 has not been well characterized.

In this study, we determined that the epitope of the V9 $\mathrm{mAb}$ corresponds to a sequence of thirteen amino acids in the C-terminal region of vimentin, within which one amino acid, the asparagine at position 417 , is critical for binding to the $\mathrm{mAb}$. This report is the first regarding precise determination of the epitope of the potent antibody V9 and these results will lead to the development of assays with high specificity for the detection of vimentin and thereby facilitate the diagnosis of malignant tumors.

\section{Materials and methods}

Antibodies. The following commercial antibodies were used: Mouse monoclonal anti-vimentin (V9, Dako, Tokyo, Japan) and anti- $\beta$-actin (AC-15, Sigma, Tokyo, Japan); rabbit polyclonal anti-vimentin (SC-5565, Santa Cruz Biotechnology, Dallas, USA) and anti-GST (60-021, BioAcademia, Osaka, Japan); horseradish peroxidase (HRP)-conjugated goat $\mathrm{F}\left(\mathrm{ab}^{\prime}\right)_{2}$ anti-mouse (710-1332, Rockland Immunochemicals, Limerick, USA) and goat anti-rabbit IgG (111-035-003, Jackson ImmunoResearch Laboratories, West Grove, USA).

Cell culture. The MIA PaCa-2 human pancreatic cancer cell line JCRB0070 and the mouse embryonic fibroblast NIH3T3 cell line JCRB1503 were purchased from the Japanese Collection of Research Bioresources Cell Bank (Osaka, Japan). The mouse fibroblast L cell line CRL-2648 was obtained from the American Tissue Culture Center (Manassas, USA). To maintain authenticity of the cell lines, multiple aliquots of frozen stocks were prepared from initial stocks, and every 3 months, a new frozen stock was used for the experiments. The cells were routinely inspected for identity by morphology and growth curve analysis and were validated to be mycoplasma free. The cells were cultured in DMEM medium containing 10\% fetal bovine serum and were maintained at $37^{\circ} \mathrm{C}$ in a humidified atmosphere with $5 \% \mathrm{CO}_{2}$.

Gene cloning. Full-length and truncated mutants of the vimentin protein were prepared by polymerase chain reaction (PCR) amplification using the Pfu polymerase (BioAcademia) and HeLa-derived cDNA (full-length) or the full-length human vimentin cDNA (truncated mutants) as the template. The primers used, containing BamHI and EcoRI restriction sites as indicated (underlines), were 5'-GGGGATCCTCCACCAGGT CCGTGTCCTCG-3' and 5'-GGGAATTCTTATTCAAGG TCATCGTGATGCTG-3' for full-length vimentin (amino acids 2 to 466); 5'-GGGGATCCTCCACCAGGTCCGTGTCCT CG-3' and 5'-GGGAATTCTTAGGCAGCCGTGAGGTCA GGCTT-3' for vim-NT (amino acids 2 to 268); 5'-GGGGAT CCGCAGCCATGGACAAAGCCCGCGTCGAGGT-3' and 5'-GGGAATTCTTATTCAAGGTCATCGTGATGCTG-3' for vim-CT (amino acids 167 to 466); 5'-GGGGATCCAACACCC TGCAATCTTTCAG-3' and 5'-GGGAATTCTTAGCGCAGG GCGTCATTGTTCCG-3' for vim-CT1 (amino acids 201 to
310); 5'-GGGGATCCGCTGCCAACCGGAACAATGAC-3' and 5'-GGGAATTCTTACAGCAGCTTCCTGTAGGTGG-3' for vim-CT2 (amino acids 301 to 404); and 5'-GGGGATCCG CCCTTGACATTGAGATTGCC-3' and 5'-GGGAATTCT TATTCAAGGTCATCGTGATGCTG-3' for vim-CT3 (amino acids 392 to 466). The amplified fragments were digested with BamHI and EcoRI and cloned into the pGEX-4T2 vector (GE Healthcare, Buckinghamshire, England) digested with the same two enzymes. BL21 bacteria were transformed with the plasmids, and protein expression was induced with $1 \mathrm{mM}$ isopr opyl- $\beta$-D-1-thiogalactopyranoside (IPTG). The final constructs were sequenced to ensure that no mutations had occurred during the PCR and cloning processes.

The point mutation construct $(\mathrm{N} 417 \mathrm{~T})$ was generated using the PCR overlap extension method. Two reactions were performed in the first round of PCR using full-length vimentin as a template and the following primer pairs, containing BamHI and EcoRI restriction sites as indicated (underlines) 5'-GGGGATCCTCCACCAGGTCCGTGTCCT CG-3' and 5'-GGTTCAGGGAGGAAAAGGTTGGAAGAG GCAGAGA-3'; and 5'-TCTCTGCCTCTTCCAACCTTTTCC TCCCTGAAC-3' and 5'-GGGAATTCTTATTCAAGGTCA TCGTGATGCTG-3'. The two purified PCR products were mixed with the primer pair 5'-GGGGATCCTCCACCAGGT CCGTGTCCTCG-3' and 5'-GGGAATTCTTATTCAAGG TCATCGTGATGCTG-3' for the second round of PCR. The PCR product was cloned into the BamHI/EcoRI sites of the pGEX-4T2 vector for expression in E. coli. The target mutation was identified by sequence analysis of the region of the mutation.

All short vimentin fragments were generated by annealing complementary oligos. The oligos used to synthesize the indicated fragments were: 5'-GATCCATTTCTCTGCCTCTTC CAAACTTTTCCTCCCTGAACCTGTAAG-3' and 5'-AAT TCTTACAGGTTCAGGGAGGAAAAGTTTGGAAGAGGC AGAGAAATG-3' (vim 411-423), 5'-GATCCATTTCTCT GCCTCTTCCAACATTTTCCTCCCTGAACCTGTAAG-3' and 5'-AATTCTTACAGGTTCAGGGAGGAAAATGTT GGAAGAGGCAGAGAAATG-3' (vim 411-423_N417T), 5'-GATCCTCTCTGCCTCTTCCAAACTTTTCCTCCCTGA ACTAAG-3' and 5'-AATTCTTAGTTCAGGGAGGAAAAG TTTGGA AGAGGCAGAGAG-3' (vim 412-422), 5'-GAT CCCTGCCTCTTCCAAACTTTTCCTCCCTGTAAG-3' and 5'-AАтTCTTACAGGGAGGAAAAGTTTGGAAGAGGC AGG-3' (vim 413-421). Annealing reactions containing equal molar amounts of each oligo were performed in the thermal cycler as follows: Initial denaturation at $98^{\circ} \mathrm{C}$ for $3 \mathrm{~min}$ followed by a temperature decrease of $2^{\circ} \mathrm{C}$ every $20 \mathrm{~s}$ down to $4^{\circ} \mathrm{C}$ to allow the oligos to anneal slowly. The oligos contained the BamHI/EcoRI restriction sites for cloning of the vimentin fragments into these restriction sites within the pGEX-4T2 vector.

Western blotanalysis. The total protein samples from cell lysates after IPTG induction were mixed with SDS buffer $(125 \mathrm{mM}$ Tris-HCl, pH 6.8, 5\% glycerol, 4\% SDS, $0.005 \%$ bromophenol blue, and 10\% 2-mercaptoethanol). Extracts were subjected to $12 \%$ SDS-polyacrylamide gel electrophoresis and transferred onto a polyvinylidene difluoride membrane (Merck Millipore, Darmstadt, Germany). After blocking with 5\% nonfat dry milk for $30 \mathrm{~min}$, the membranes were incubated with the indicated 
A

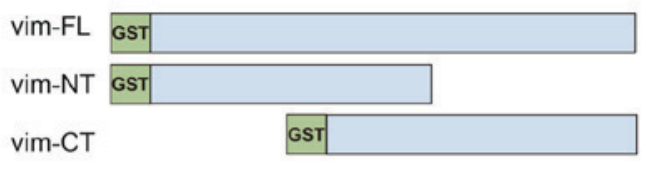

B

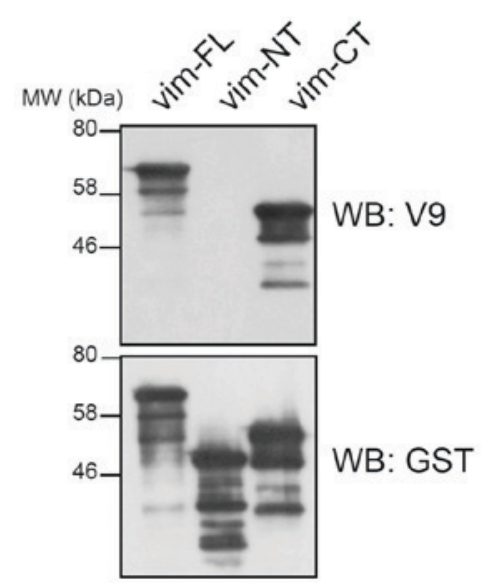

C

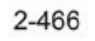

$2-268$

$167-466$

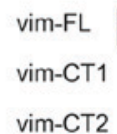

os

vim-CT3

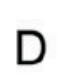

Figure 1. Analysis of the reactivity of the mouse anti-human vimentin monoclonal antibody V9 with vimentin truncation mutants. (A) Schematic representation of full-length and truncated mutants of human vimentin. Vim-FL, full-length (2-466); vim-NT, N-terminal (2-268); vim-CT, C-terminal (167-466) vimentin. All constructs were GST-tagged at the N-terminus. (B) Western blot analysis (WB) using anti-vimentin (V9) or anti-GST antibodies. The proteins were expressed in E. coli using the pGEX-4T2 vector. (C) Schematic representation of full-length (vim-FL) and truncated mutants, vim-CT1 (201-310), vim-CT2 (301-404) and vim-CT3 (392-466), of human vimentin. (D) Western blot analysis of bacterially expressed GST-tagged full-length or truncated mutants of vimentin with the indicated antibodies.

antibodies for 1 hat room temperature followed by incubation with secondary antibodies (HRP-conjugated anti-mouse or anti-rabbit IgG at the appropriate dilutions) for $45 \mathrm{~min}$ at room temperature. Antibody binding was detected with an enhanced chemiluminescence detection system (PerkinElmer, Waltham, USA).

Bioinformatics. Sequences were aligned with Clustal Omega (http://www.ebi.ac.uk/Tools/msa/clustalo/).

\section{Results}

The V9 mAb recognized the C-terminal region of vimentin ( a a 392-466). To map the epitope recognized by the V9 antibody, we first generated recombinant N-terminal (aa 2-268) or C-terminal (aa 167-466) vimentin protein fragments fused with GST (Fig. 1A). Western blot analysis showed that the V9 $\mathrm{mAb}$ bound to the $\mathrm{C}$-terminal but not to the $\mathrm{N}$-terminal vimentin fragment (Fig. 1B), confirming previous reports $(16,17)$. To further determine the precise location of the V9 mAb epitope, we constructed three GST-tagged C-terminal truncated mutants of vimentin, vim-CT1 (aa 201-310), vim-CT2 (aa 301-404) and vim-CT3 (aa 392-466) (Fig. 1C). These mutants were expressed in E. coli and their reactivity with the $\mathrm{V} 9 \mathrm{mAb}$ was examined by Western blotting. Of these mutants only vim-CT3 was recognized by the V9 $\mathrm{mAb}$; neither vim-CT1 nor vim-CT2 reacted with the $\mathrm{V} 9 \mathrm{mAb}$ (Fig. 1D). These results suggested that the epitope recognized by the $\mathrm{V} 9 \mathrm{mAb}$ is located within amino acid residues $392-466$ of the vimentin protein.

Asparagine at position 417 was indispensable for detection of vimentin by the $V 9 m A b$. The antibody V9 was shown to react with the vimentin of various mammalian species, including human, hamster, rat, and pig (18), but it did not detect vimentin in mouse cells or chicken fibroblasts (18). To confirm this observation, we first examined whether the V9 $\mathrm{mAb}$ could recognize vimentin in the mouse fibroblast cell line, NIH 3T3, which normally expresses endogenous vimentin (19). Western blot analysis of vimentin in human (MIA PaCa-2) and mouse (NIH 3T3) cell lines confirmed that the $\mathrm{V} 9 \mathrm{mAb}$ recognizes the human but not the mouse vimentin protein (Fig. 2A). Similar results were obtained using another mouse L fibroblast cell line, in which expression of mouse vimentin of the cell line was detected with a rabbit anti-vimentin polyclonal antibody (Fig. 2B). To investigate the mechanism behind the inability of the V9 $\mathrm{mAb}$ to recognize vimentin in mouse cells, we compared the amino acid residues (residues 392-466) flanking the epitope among different species (Fig. 2C). This sequence comparison showed that most of the amino acid residues were conserved among the species analyzed. However, one amino acid that was unique to mouse was observed at position 417 ; mouse vimentin contains threonine at position 417 , whereas human, hamster, rat, and pig have an asparagine residue at the corresponding position. We therefore hypothesized that the inability of the $\mathrm{V} 9 \mathrm{mAb}$ to recognize mouse vimentin was probably attributable to the variation at position 417 . To test this hypothesis, we generated an expression construct in which a single amino acid substitution, that of threonine for asparagine at position 417, was introduced into the full-length GST-tagged human vimentin protein. The plasmid construct was verified by DNA sequence analysis and the recombinant protein was expressed in E. coli BL21 cells. Western blot analysis showed that the mutation N417T completely inhibited V9 antibody binding (Fig. 2D). These results indicate 


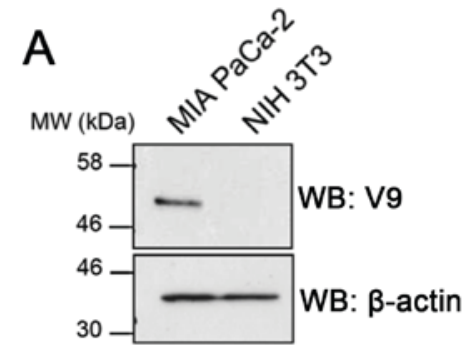

C 392

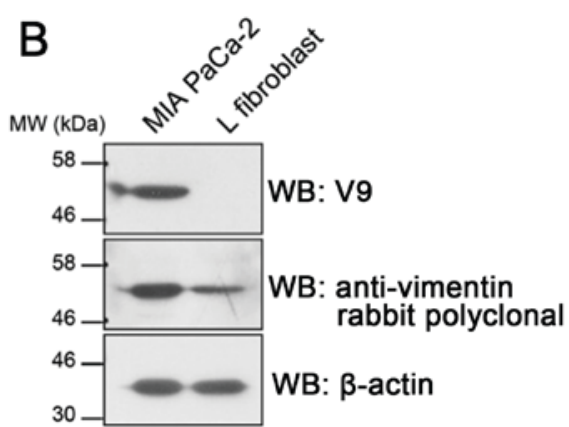

417

human ALDIEIATYRKLLEGEESRISLPLPNNFSLNLRETNLD mouse ALDIEIATYRKLLEGEESRISLPLPTFSSLNLRETNLE hamster ALDIEIATYRKLLEGEESRISLPLPNFSSLNLRETNLE rat ALDIEIATYRKLLEGEESRISLPLPNFSSLNLRETNLE pig ALDIEIATYRKLLEGEESRISLPLPNFSSLNLRETNLE

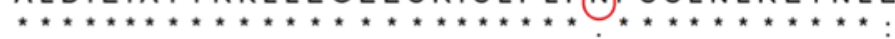

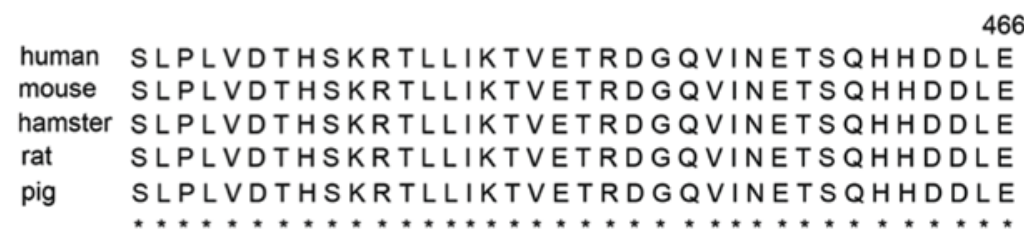

D

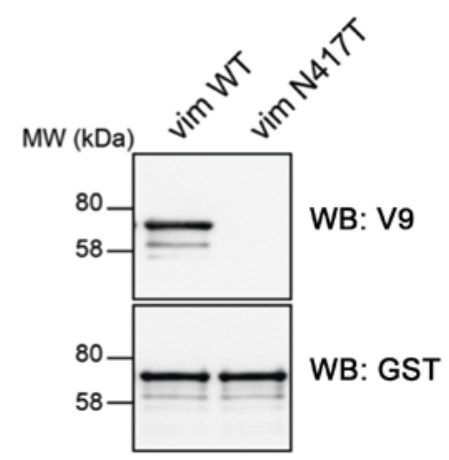

Figure 2. Asparagine at position 417 of human vimentin is critical for $\mathrm{V} 9 \mathrm{mAb}$ recognition of vimentin. (A and B) Whole cell extracts from MIA PaCa-2 (human pancreatic cancer cell line) and mouse NIH3T3 and L fibroblast cell lines were analyzed for vimentin expression by Western blotting with the indicated antibodies. $\beta$-actin was used as a loading control. (C) Amino acid sequence alignment of the C-terminal region of vimentin (residues 392-466) from different species. The red box shows the only amino acid difference between the sequence of mouse vimentin and that of human, hamster, rat, and pig. Multiple sequence alignment was performed using Clustal Omega software. (D) Western blot analysis of bacterially expressed GST-tagged wild-type and mutant (N417T) human full-length vimentin with the indicated antibodies.

that the asparagine residue at position 417 is a critical residue for V9 antibody binding and that its replacement with threonine at position 417 effectively inhibited this interaction.

Precise determination of the epitope for the V9 $m A b$. To precisely define the amino acid residues that are involved in V9 binding, we constructed a set of GST-tagged vimentin fragments with amino acid sequences containing the asparagine at position 417 that was implicated as being essential for binding based on our above results. These truncated peptide fragments were expressed and analyzed for their reactivity with the $\mathrm{V} 9 \mathrm{mAb}$ by Western blotting. The blotting showed that the $\mathrm{V} 9 \mathrm{mAb}$ could only recognize the peptide vim 411-423 ( ${ }^{411}$ ISLPLPNFSSLNL $^{423}$ ) (Fig. 3). As expected, asparagine to threonine substitution at position 417 (vim 411-423_N417T) abolished V9 mAb binding. Neither of the peptides vim 412-422 $\left({ }^{412}\right.$ SLPLPNFSSLN $\left.^{422}\right)$ or vim 413-421 ( ${ }^{413}$ LPLPNFSSL $\left.^{421}\right)$ were recognized by the V9

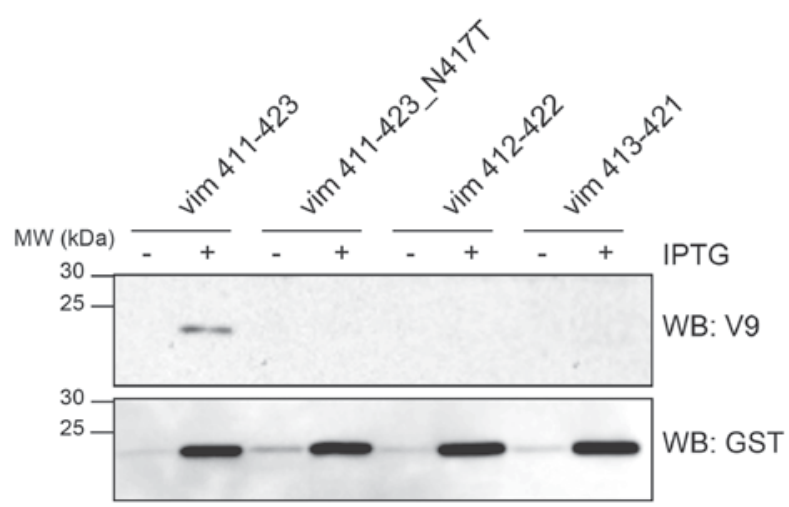

Figure 3. The monoclonal antibody V9 epitope in human vimentin corresponds to amino acid residues 411 to 423 . Western blot analysis of GST-tagged bacterially expressed human vimentin truncated fragments and an N417T substitution truncated fragment (411-423, 411-423_N417T, 412-422 and 413-421) with the indicated antibodies. Proteins were extracted from $E$. coli transformed with the pGEX-4T2 constructs after one-hour induction with (+) or without (-) IPTG. Equal amounts of total protein $(10 \mu \mathrm{g})$ were loaded in each lane. 
mAb. We concluded that the thirteen amino acid residues ${ }^{411}$ ISLPLPNFSSLNL ${ }^{423}$ in vimentin represent the minimal requirement for recognition of the epitope by the V9 mAb.

\section{Discussion}

Vimentin is associated with cancer invasion and poor prognosis in many types of cancers (9) and is therefore a potent target for cancer therapy. Recent studies demonstrated that vimentin, which is located intracellularly in normal cells, is exposed on the surface of malignant cells $(20,21)$. Using the vimentin-specific mAb V9, Steinmetz et al showed that vimentin is present on the surface of metastatic prostate cancer cells, suggesting that vimentin might be a useful target for antibody-based therapy (17). To our knowledge, there have been no previous reports of epitope mapping of the V9 mAb.

Here, we have characterized the epitope recognized by the V9 mAb by testing its reactivity with cell extracts expressing various deletion mutants of the human vimentin protein. We determined that the $\mathrm{V} 9 \mathrm{mAb}$ reacts with residues 392-466, which are at the $\mathrm{C}$-terminal end of the vimentin molecule (Fig. 1C). The antibody shows interspecies cross-reactivity recognizing human vimentin as well as vimentin from monkey, dog, horse, cattle, rabbit, rat, hamster, and pig (18), which is consistent with the finding that the amino acid sequence of the epitope region is highly conserved among these diverse species (Fig. 2C). However, there is one amino acid difference at position 417 within these 392-466 residues that differs between the mouse vimentin and the vimentin of other species. At this position, the asparagine residue in human, hamster, rat, and pig is replaced by threonine in the mouse vimentin. Consistent with a previous report (18), the $\mathrm{V} 9 \mathrm{mAb}$ did not recognize vimentin in mouse NIH 3 T3 and L fibroblast cell lines (Fig. 2A, B), suggesting that this single amino acid substitution at position 417 of mouse vimentin might prevent binding of the V9 antibody. In agreement with this possibility, the single amino acid replacement of asparagine at position 417 with threonine in full-length human vimentin (vim N417T) completely abrogated the reactivity of the $\mathrm{V} 9 \mathrm{mAb}$, as shown in Fig. 2D.

To study the specificity of V9 mAb binding in more detail and to map the epitope recognized by the V9 mAb, we generated a series of fragments of the vimentin protein and used them to screen for the minimal epitope by Western blotting. We found that the thirteen amino acid sequence ${ }^{411}$ ISLPLPNFSSLNL ${ }^{423}$ is the minimal recognition sequence for $\mathrm{V} 9 \mathrm{mAb}$ binding and that substitution of asparagine at position 417 with threonine, as is found in mouse vimentin, inhibited binding by the antibody (Fig. 3). These data indicated the importance of this asparagine residue of the epitope in antibody-epitope binding reactivity.

In summary, our results more precisely define the epitope in vimentin that is recognized by the V9 $\mathrm{mAb}$. The information provided in this study will facilitate the development of specific diagnostic assays for cancer therapeutics.

\section{Acknowledgements}

We acknowledge the technical expertise of the Center for Integrated Research in Science at Shimane University. We also thank Ms. Yuko Fukuma for technical assistance. This work was supported by the SUIGAN project, Shimane University, Japan.

\section{References}

1. Steinert PM and Roop DR: Molecular and cellular biology of intermediate filaments. Annu Rev Biochem 57: 593-625, 1988.

2. Ivaska J, Pallari HM, Nevo J and Eriksson JE: Novel functions of vimentin in cell adhesion, migration, and signaling. Exp Cell Res 313: 2050-2062, 2007.

3. Fuchs E and Weber K: Intermediate filaments: Structure, dynamics, function, and disease. Annu Rev Biochem 63: 345-382, 1994.

4. Parry DA: Microdissection of the sequence and structure of intermediate filament chains. Adv Protein Chem 70: 113-142, 2005.

5. Sihag RK, Inagaki M, Yamaguchi T, Shea TB and Pant HC: Role of phosphorylation on the structural dynamics and function of types III and IV intermediate filaments. Exp Cell Res 313: 2098-2109, 2007.

6. Matsuyama M, Tanaka H, Inoko A, Goto H, Yonemura S, Kobori K, Hayashi Y, Kondo E, Itohara S, Izawa I and Inagaki M: Defect of mitotic vimentin phosphorylation causes microophthalmia and cataract via aneuploidy and senescence in lens epithelial cells. J Biol Chem 288: 35626-35635, 2013.

7. Thiery JP: Epithelial-mesenchymal transitions in tumour progression. Nat Rev Cancer 2: 442-454, 2002.

8. Zhu QS, Rosenblatt K, Huang KL, Lahat G, Brobey R, Bolshakov S, Nguyen T, Ding Z, Belousov R, Bill K, et al: Vimentin is a novel AKT1 target mediating motility and invasion. Oncogene 30: 457-470, 2011.

9. Satelli A and Li S: Vimentin in cancer and its potential as a molecular target for cancer therapy. Cell Mol Life Sci 68: 3033-3046, 2011

10. Gilles C, Polette M, Zahm JM, Tournier JM, Volders L, Foidart JM and Birembaut P: Vimentin contributes to human mammary epithelial cell migration. J Cell Sci 112: 4615-4625, 1999.

11. Singh S, Sadacharan S, Su S, Belldegrun A, Persad S and Singh G: Overexpression of vimentin: Role in the invasive phenotype in an androgen-independent model of prostate cancer. Cancer Res 63: 2306-2311, 2003.

12. Sun S, Poon RT, Lee NP, Yeung C, Chan KL, Ng IO, Day PJ and Luk JM: Proteomics of hepatocellular carcinoma: Serum vimentin as a surrogate marker for small tumors $(<\mathrm{or}=2 \mathrm{~cm})$. J Proteome Res 9: 1923-1930, 2010.

13. Bukhari S, Mokhdomi TA, Chikan NA, Amin A, Qazi H, Wani SH, Wafai AH, Tyub S, Mustafa F, Mir MS, et al: Affinity proteomics led identification of vimentin as a potential biomarker in colon cancers: Insights from serological screening and computational modelling. Mol Biosyst 11: 159-169, 2015.

14. Boilard E, Bourgoin SG, Bernatchez C and Surette ME: Identification of an autoantigen on the surface of apoptotic human $\mathrm{T}$ cells as a new protein interacting with inflammatory group IIA phospholipase A2. Blood 102: 2901-2909, 2003.

15. Osborn M, Debus E and Weber K: Monoclonal antibodies specific for vimentin. Eur J Cell Biol 34: 137-143, 1984.

16. Moisan E and Girard D: Cell surface expression of intermediate filament proteins vimentin and lamin B1 in human neutrophil spontaneous apoptosis. J Leukoc Biol 79: 489-498, 2006.

17. Steinmetz NF, Maurer J, Sheng H, Bensussan A, Maricic I, Kumar V and Braciak TA: Two domains of vimentin are expressed on the surface of lymph node, bone and brain metastatic prostate cancer lines along with the putative stem cell marker proteins CD44 and CD133. Cancers (Basel) 3: 2870-2885, 2011.

18. Bohn W, Wiegers W, Beuttenmüller $M$ and Traub P: Species-specific recognition patterns of monoclonal antibodies directed against vimentin. Exp Cell Res 201: 1-7, 1992.

19. Yates B, Zetterberg C, Rajeev V, Reiss M and Rittling SR: Promoter-independent regulation of vimentin expression in mammary epithelial cells by val(12)ras and TGFbeta. Exp Cell Res 313: 3718-3728, 2007.

20. Satelli A, Mitra A, Cutrera JJ, Devarie M, Xia X, Ingram DR, Dibra D, Somaiah N, Torres KE, Ravi V, et al: Universal marker and detection tool for human sarcoma circulating tumor cells. Cancer Res 74: 1645-1650, 2014.

21. Satelli A, Brownlee Z, Mitra A, Meng QH and Li S: Circulating tumor cell enumeration with a combination of epithelial cell adhesion molecule- and cell-surface vimentin-based methods for monitoring breast cancer therapeutic response. Clin Chem 61: 259-266, 2015. 